\title{
spinifex: An R Package for Creating a Manual Tour of Low-dimensional Projections of Multivariate Data
}

\author{
by Nicholas Spyrison and Dianne Cook
}

\begin{abstract}
Dynamic low-dimensional linear projections of multivariate data collectively known as tours provide an important tool for exploring multivariate data and models. The $\mathrm{R}$ package tourr provides functions for several types of tours: grand, guided, little, local and frozen. Each of these can be viewed dynamically, or saved into a data object for animation. This paper describes a new package, spinifex, which provides a manual tour of multivariate data where the projection coefficient of a single variable is controlled. The variable is rotated fully into the projection, or completely out of the projection. The resulting sequence of projections can be displayed as an animation, with functions from either the plotly or gganimate packages. By varying the coefficient of a single variable, it is possible to explore the sensitivity of structure in the projection to that variable. This is particularly useful when used with a projection pursuit guided tour to simplify and understand the solution. The use of the manual tour is applied particle physics data to illustrate the sensitivity of structure in a projection to specific variable contributions.
\end{abstract}

\section{Introduction}

Exploring multivariate spaces is a challenging task, increasingly so as dimensionality increases. Traditionally, static low-dimensional projections are used to display multivariate data in two dimensions including principal component analysis, linear discriminant spaces or projection pursuit. These are useful for finding relationships between multiple variables, but they are limited because they show only a glimpse of the high-dimensional space. An alternative approach is to use a tour (Asimov, 1985) of dynamic linear projections to look at many different low-dimensional projections. Tours can be considered to extend the dimensionality of visualization, which is important as data and models exist in more than 3D. The package tourr (Wickham et al., 2011) provides a platform for generating tours. It can produce a variety of tours, each paired with a variety of possible displays. A user can make a grand, guided, little, local or frozen tour, and display the resulting projected data as a scatterplot, density plot, histogram, or even as Chernoff faces if the projection dimension is more than 3.

This work adds a manual tour to the collection. The manual tour was described in Cook and Buja (1997) and allows a user to control the projection coefficients of a selected variable in a 2D projection. The manipulation of these coefficients allows the analyst to explore their sensitivity to the structure within the projection. As manual tours operate on only one variable at a time, they are particularly useful once a feature of interest has been identified.

One way to identify "interesting" features is with the use of a guided tour (Cook et al., 1995-09). Guided tours select a very specific path, which approaches a projection that optimizes an objective function. The optimization used to guide the tour is simulated annealing (Kirkpatrick et al., 1983). The direct optimization of a function allows guided tours to rapidly identify interesting projection features given the relatively large parameter-space. After a projection of interest is identified, an analyst can then use the "finer brush" of the manual tour to control the contributions of individual variables to explore the sensitivity they have on the structure visible in the projection.

The paper is organized as follows. Section 2.2 describes the algorithm used to perform a radial manual tour as implemented in the package spinifex. Section 2.2.2 explains how to generate an animation of the manual tour using the animation frameworks offered by plotly (Sievert, 2020) and gganimate (Pedersen and Robinson, 2020). Package functionality and code usage following the order applied in the algorithm follows in section 2.3.3. Section 2.4 illustrates how this can be used for sensitivity analysis applied to multivariate data collected on high energy physics experiments (Wang et al., 2018). Section 2.5 summarizes this paper and discusses potential future directions.

\section{Algorithm}

The algorithm to conduct a manual tour interactively, by recording mouse/cursor motion, is described in detail in Cook and Buja (1997). Movement can be in any direction and magnitude, but it can also be constrained in several ways: 
- radial: fix the direction of contribution, and allow the magnitude to change.

- angular: fix the magnitude, and allow the angle or direction of the contribution to vary.

- horizontal, vertical: allow rotation only around the horizontal or vertical axis of the current 2D projection.

The algorithm described here produces a radial tour as an animation sequence. It takes the current contribution of the chosen variable, and using rotation brings this variable fully into the projection, completely removes it, and then returns to the original position.

\section{Notation}

The notation used to describe the algorithm for a 2D radial manual tour is as follows:

- $\mathbf{X}$, the data, an $n \times p$ numeric matrix to be projected to 2D.

- $\mathbf{B}=\left(B_{1}, B_{2}\right)$, any $2 \mathrm{D}$ orthonormal projection basis, $p \times 2$ matrix, describing the projection from $\mathbb{R}^{p} \Rightarrow \mathbb{R}^{2}$. This is called this the "original projection" because it is the starting point for the manual tour.

- $k$, is the index of the variable to manipulate, called the "manip var".

- e, a 1D basis vector of length $p$, with 1 in the $k$-th position and 0 elsewhere.

- $\mathbf{M}$ is a $p \times 3$ matrix, defining the $3 \mathrm{D}$ subspace where data rotation occurs and is called the manip(ulation) space.

- $\mathbf{R}$, the 3D rotation matrix, for performing unconstrained 3D rotations within the manip space, M.

- $\theta$, the angle of in-projection rotation, for example, on the reference axes; $c_{\theta}, s_{\theta}$ are its cosine and sine.

- $\phi$, the angle of out-of-projection rotation, into the manip space; $c_{\phi}, s_{\phi}$ are its cosine and sine. The initial value for animation purposes is $\phi_{1}$.

- $\mathbf{U}$, the axis of rotation for out-of-projection rotation orthogonal to e.

- $\mathbf{Y}$, the resulting projection of the data through the manip space, $\mathbf{M}$, and rotation matrix, $\mathbf{R}$.

The algorithm operates entirely on projection bases and incorporates the data only when making the projected data plots, in light of efficiency.

\section{Steps}

\section{Step 0) Set up}

The flea data (Lubischew (1962)), available in the tourr package, is used to illustrate the algorithm. The data contains 74 observations on 6 variables, which are physical measurements made on flea beetles. Each observation belongs to one of three species.

An initial 2D projection basis must be provided. A suggested way to start is to identify an interesting projection using a projection pursuit guided tour. Here the holes index is used to find a 2D projection of the flea data, which shows three separated species groups. Figure 1 shows the initial projection of the data. The left panel displays the projection basis (B) and can be used as a visual guide of the magnitude and direction that each variable contributes to the projection. The right panel shows the projected data, $\mathbf{Y}_{[n, 2]}=\mathbf{X}_{[n, p]} \mathbf{B}_{[p, 2]}$. The color and shape of points are mapped to the flea species. This plot is made using the view_basis () function in spinifex, which generates a ggplot2 (Wickham, 2016) object.

\section{Step 1) Choose manip variable}

In figure 1 the contribution of the variables tars 1 and aede 2 mostly contrast the contribution of the other four variables. These two variables combined contribute in the direction of the projection where the purple cluster is separated from the other two clusters. The variable aede 2 is selected as the manip var, the variable to be controlled in the tour. The question that will be explored is: how important is this variable to the separation of the clusters in this projection? 


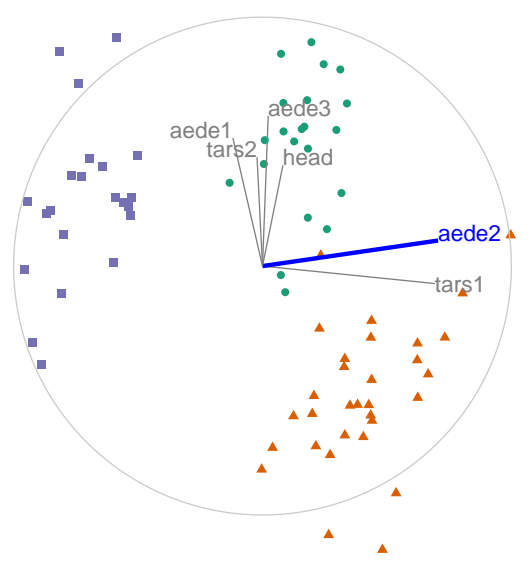

Figure 1: Initial 2D projection: representation of the basis (left) and resulting data projection (right) of standardized flea data. The color and shape of data points are mapped to beetle species. The basis was identified using a projection pursuit guided tour, with the holes index. The contribution of the variables aede 2 and tars 1 approximately contrasts the other variables. The visible structure in the projection are the three clusters corresponding to the three species. Produced with the function view_basis().

\section{Step 2) Create the 3D manip space}

Initialize the coordinate basis vector as a zero vector, $\mathbf{e}$, of length $p$, and set the $k$-th element to 1 . In the example data, aede2 is the fifth variable in the data, so $k=5$, set $e_{5}=1$. Use a Gram-Schmidt process to orthonormalize the coordinate basis vector on the original 2D projection to describe a 3D manip space, $\mathbf{M}$.

$$
\begin{aligned}
e_{k} & \leftarrow 1 \\
\mathbf{e}_{[p, 1]}^{*} & =\mathbf{e}-\left\langle\mathbf{e}, \mathbf{B}_{1}\right\rangle \mathbf{B}_{1}-\left\langle\mathbf{e}, \mathbf{B}_{2}\right\rangle \mathbf{B}_{2} \\
\mathbf{M}_{[p, 3]} & =\left(\mathbf{B}_{1}, \mathbf{B}_{2}, \mathbf{e}^{*}\right)
\end{aligned}
$$

The manip space provides a 3D projection from $p$-dimensional space, where the coefficient of the manip var can range completely between $[0,1]$. This $3 \mathrm{D}$ space serves as the medium to rotate the projection basis relative to the selected manipulation variable. Figure 2 illustrates this 3D manip space with the manip var highlighted. This representation is produced by calling the view_manip_space() function. This diagram is purely used to help explain the algorithm.

\section{Step 3) Defining a 3D rotation}

The basis vector corresponding to the manip var (red line in Figure 2), can be operated like a lever anchored to the origin. This is the process of the manual control, that rotates the manip variable into and out of the 2D projection (Figure 3). As the variable contribution is controlled, the manip space rotates, and the projection onto the horizontal projection plane correspondingly changes. This is a manual tour. Generating a sequence of values for the rotation angles produces a path for the rotation of the manip space.

For a radial tour, fix $\theta$, the angle describing rotation within the projection plane, and compute a sequence for $\phi$, defining movement out of the plane. This will change $\phi$ from the initial value, $\phi_{1}$, the angle between $\mathbf{e}$ and its shadow in $\mathbf{B}$, to a maximum of 0 (manip var fully in projection), then to a minimum of $\pi / 2$ (manip var out of projection), before returning to $\phi_{1}$.

Rotations in $3 \mathrm{D}$ can be defined by the axes they pivot on. Rotation within the projection, $\theta$, is rotation around the $Z$ axis. Out-of-projection rotation, $\phi$, is the rotation around an axis on the $X Y$ plane, $\mathbf{U}$, orthogonal to e. Given these axes, the rotation matrix, $\mathbf{R}$ can be written as follows, using Rodrigues' rotation formula (originally published in Rodrigues (1840)): 


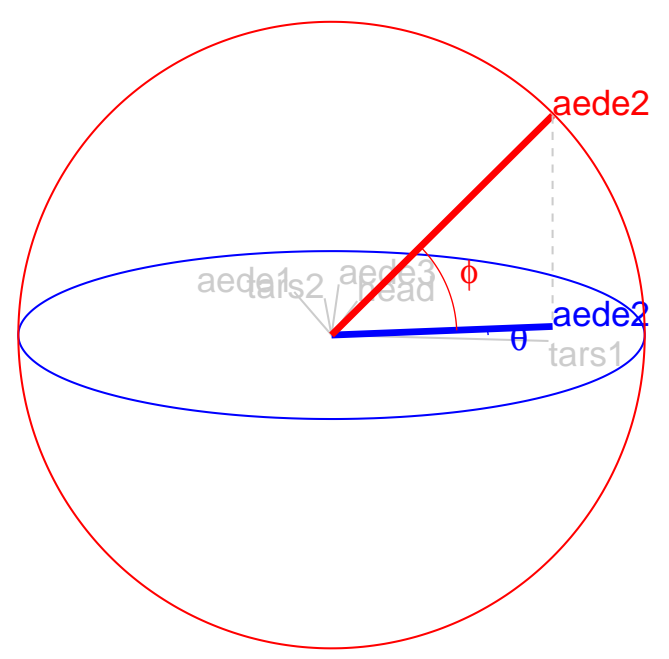

Figure 2: Illustration of a 3D manip space, this space is rotated effectively changing the contribution of the manip variable, aede2 in the example data. The blue circle and variable map lies on the projection plane. The red circle, orthogonal to the projection plane, illustrates the manipulation space and how the manip var can be controlled and how this affects the variable contribution back onto the projection plane. The other variables are omitted from the manipulation dimension for simplicity. Picturing the other variables in that dimension reveals the intuition that rotating one variable performs a constrained rotation on the others. This is illustrated with the view_manip_space() function.

$$
\begin{aligned}
& \mathbf{R}_{[3,3]}=\mathbf{I}_{3}+s_{\phi} \mathbf{U}+\left(1-c_{\phi}\right) \mathbf{U}^{2} \\
& =\left[\begin{array}{lll}
1 & 0 & 0 \\
0 & 1 & 0 \\
0 & 0 & 1
\end{array}\right]+\left[\begin{array}{ccc}
0 & 0 & c_{\theta} s_{\phi} \\
0 & 0 & s_{\theta} s_{\phi} \\
-c_{\theta} s_{\phi} & -s_{\theta} s_{\phi} & 0
\end{array}\right]+\left[\begin{array}{ccc}
-c_{\theta}\left(1-c_{\phi}\right) & s_{\theta}^{2}\left(1-c_{\phi}\right) & 0 \\
-c_{\theta} s_{\theta}\left(1-c_{\phi}\right) & -s_{\theta}^{2}\left(1-c_{\phi}\right) & 0 \\
0 & 0 & c_{\phi}-1
\end{array}\right] \\
& =\left[\begin{array}{ccc}
c_{\theta}^{2} c_{\phi}+s_{\theta}^{2} & -c_{\theta} s_{\theta}\left(1-c_{\phi}\right) & -c_{\theta} s_{\phi} \\
-c_{\theta} s_{\theta}\left(1-c_{\phi}\right) & s_{\theta}^{2} c_{\phi}+c_{\theta}^{2} & -s_{\theta} s_{\phi} \\
c_{\theta} s_{\phi} & s_{\theta} s_{\phi} & c_{\phi}
\end{array}\right]
\end{aligned}
$$

where

$$
\begin{aligned}
\mathbf{U} & =\left(u_{x}, u_{y}, u_{z}\right)=\left(s_{\theta},-c_{\theta}, 0\right) \\
& =\left[\begin{array}{ccc}
0 & -u_{z} & u_{y} \\
u_{z} & 0 & -u_{x} \\
-u_{y} & u_{x} & 0
\end{array}\right]=\left[\begin{array}{ccc}
0 & 0 & -c_{\theta} \\
0 & 0 & -s_{\theta} \\
c_{\theta} & s_{\theta} & 0
\end{array}\right]
\end{aligned}
$$

\section{Step 4) Creating an animation of the radial rotation}

The steps outlined above can be used to create any arbitrary rotation in the manip space. To use these for sensitivity analysis, the radial rotation is built into an animation where the manip var is rotated fully into the projection, completely out, and then back to the initial value. This involves allowing $\phi$ to vary between 0 and $\pi / 2$, call the steps $\phi_{i}$.

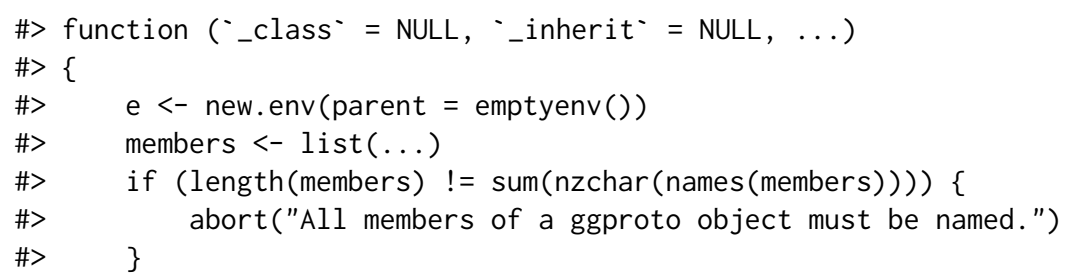


(1) phi $=0.78$

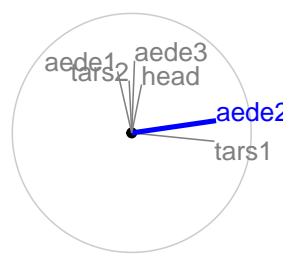

(2) $\mathrm{phi}=0$

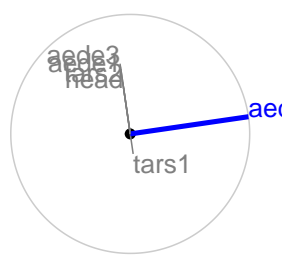

(3) phi $=1.57$

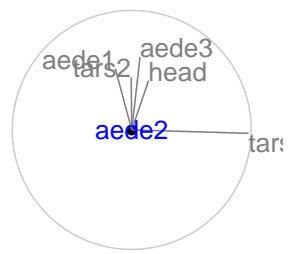

(4) phi $=0.78$

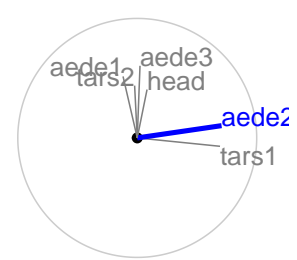

Figure 3: Snapshots of a radial manual tour manipulating aede2: (1) original projection, (2) full contribution, (3) zero contribution, (4) back to original.

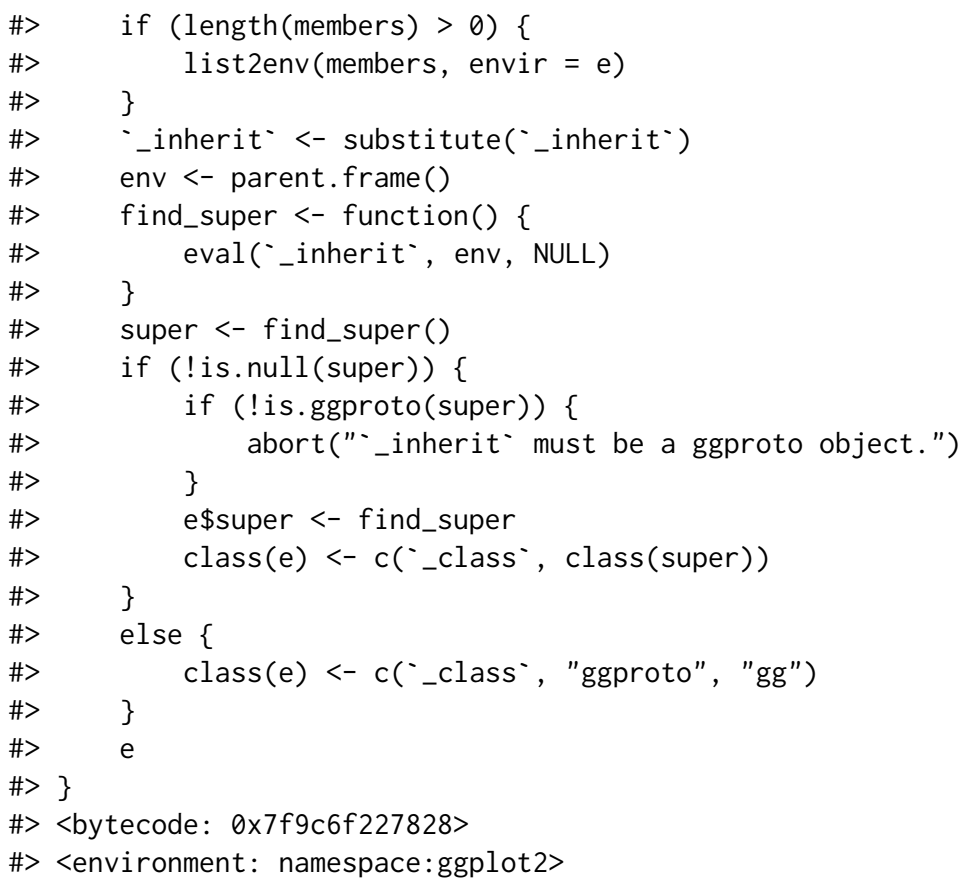

1. Set initial value of $\phi_{1}$ and $\theta: \phi_{1}=\cos ^{-1} \sqrt{B_{k 1}^{2}+B_{k 2}^{2}}, \theta=\tan ^{-1} \frac{B_{k 2}}{B_{k 1}}$. Where $\phi_{1}$ is the angle between $\mathbf{e}$ and its shadow in $\mathbf{B}$.

2. Set an angle increment $\left(\Delta_{\phi}\right)$ that sets the step size for the animation, to rotate the manip var into and out of the projection. (Note: Using angle increment, rather than a number of steps, to control the movement, is consistent with the tour algorithm as implemented in the tourr).

3. Step towards 0 , where the manip var is completely in the projection plane.

4. Step towards $\pi / 2$, where the manip variable has no contribution to the projection.

5. Step back to $\phi_{1}$.

In each of the steps 3-5, a small step may be added to ensure that the endpoints of $\phi\left(0, \pi / 2, \phi_{1}\right)$ are reached.

\section{Step 5) Projecting the data}

The operation of a manual tour is defined on the projection bases. Only when the data plot needs to be made is the data projected into the relevant basis.

$$
\mathbf{Y}_{[n, 3]}^{(i)}=\mathbf{X}_{[n, p]} \mathbf{M}_{[p, 3]} \mathbf{R}_{[3,3]}^{(i)}
$$

where $\mathbf{R}_{[3,3]}^{(i)}$ is the incremental rotation matrix, using $\phi_{i}$. To make the data plot, use the first two columns of $\mathbf{Y}$. Show the projected data for each frame in sequence to form an animation.

Figure 4 illustrates a manual tour sequence having 15 steps. The projection axes are displayed on the top half, which corresponds to the projected data in the bottom half. When aede2 is removed from 
Table 1: Summary of available functions.

\begin{tabular}{|c|c|c|}
\hline Type & Function & Description \\
\hline $\begin{array}{l}\text { construction } \\
\text { construction } \\
\text { construction }\end{array}$ & $\begin{array}{l}\text { create_manip_space } \\
\text { rotate_manip_space } \\
\text { manual_tour }\end{array}$ & $\begin{array}{l}\text { forms the 3D space of rotation } \\
\text { performs 3D rotation } \\
\text { generates sequence of } 2 D \text { frames }\end{array}$ \\
\hline $\begin{array}{l}\text { render } \\
\text { render } \\
\text { render } \\
\text { render }\end{array}$ & $\begin{array}{l}\text { array } 2 \mathrm{df} \\
\text { render_} \\
\text { render_plotly } \\
\text { render_gganimate }\end{array}$ & $\begin{array}{l}\text { turn the tour path array into long form, for plotting } \\
\text { render long form as a ggplot } 2 \text { objection for animation } \\
\text { render the animation as a plotly object (default) } \\
\text { render the animation as a gganimate object }\end{array}$ \\
\hline $\begin{array}{l}\text { animation } \\
\text { animation }\end{array}$ & $\begin{array}{l}\text { play_tour_path } \\
\text { play_manual_tour }\end{array}$ & $\begin{array}{l}\text { composite function animating the specified tour path } \\
\text { composite function animating the specified manual tour }\end{array}$ \\
\hline $\begin{array}{l}\text { specialty } \\
\text { specialty } \\
\text { specialty }\end{array}$ & $\begin{array}{l}\text { print_manip_space } \\
\text { oblique_frame } \\
\text { view_manip_space }\end{array}$ & $\begin{array}{l}\text { table of the rotated basis and manip space } \\
\text { display the reference axes of a given basis } \\
\text { illustrative display of any manip space }\end{array}$ \\
\hline
\end{tabular}

the projection, the purple cluster overlaps with the green cluster. This suggests that aede2 is important for distinguishing between these species.

Tours are typically viewed as an animation. The animation of this tour can be viewed online at https://github.com/nspyrison/spinifex_paper/blob/master/paper/gifs/flea_radialtour_mvar5. gif. The page may take a moment to load. Animations can be produced using the function play_manual_tour().

\section{Package structure and functionality}

This section describes the functions available in the package, and how to use them.

\section{Installation}

The spinifex is available from CRAN, and can be installed by:

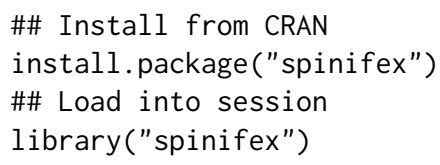

Also see the shiny app for understandign and the vignette for basic usage:

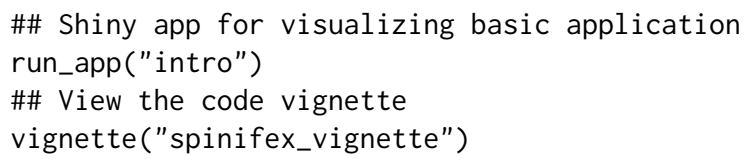

The development version can be installed from github:

\#\# Optionally install latest developmention version from GitHub remotes: : install_github("nspyrison/spinifex")

\section{Functions}

Table 1 lists the primary functions and their purpose. These are grouped into four types: construction for building a tour path, render to make the plot objects, animation for running the animation, and specialty for providing illustrations used in the algorithm description. 


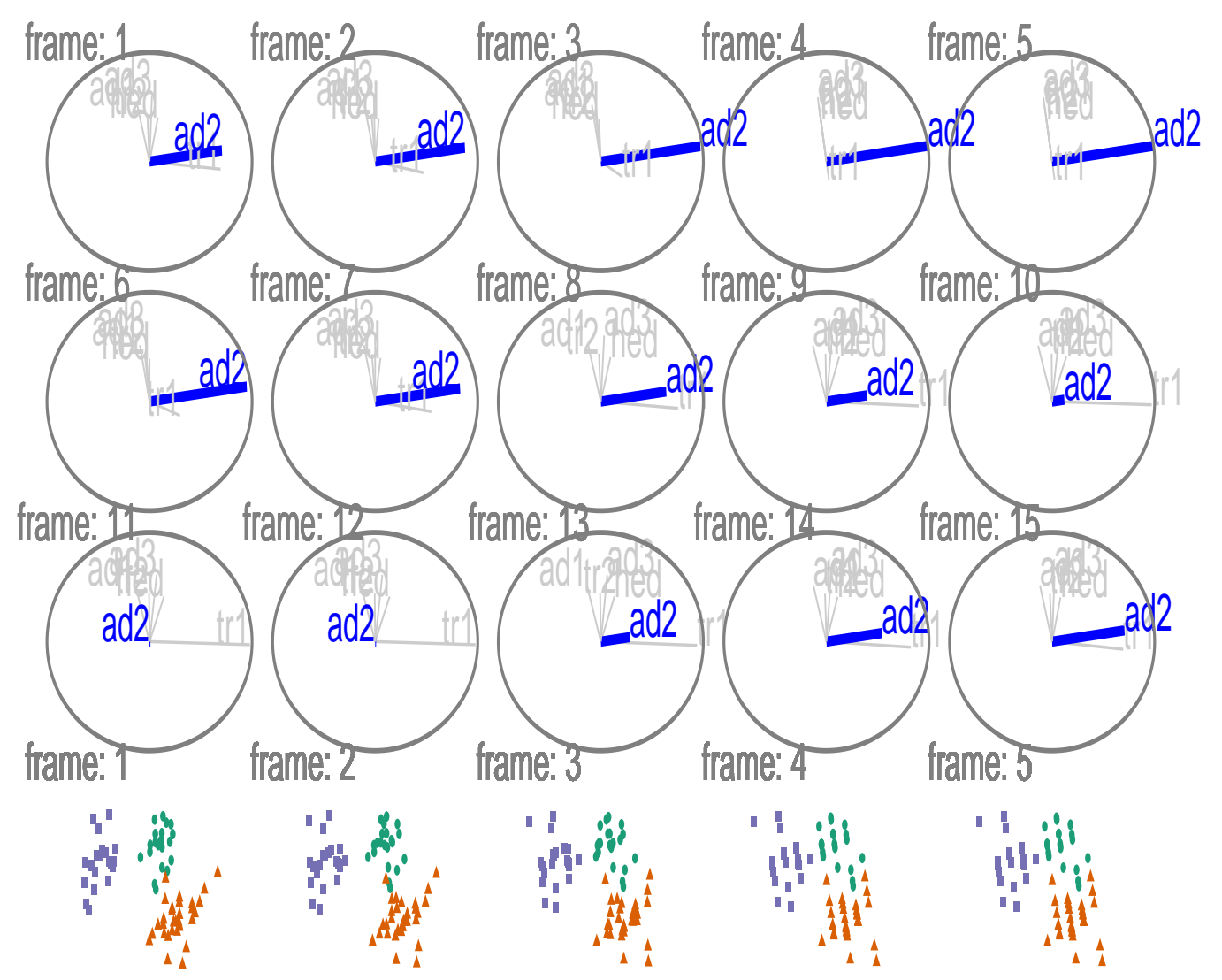

frame: 6 frame: 7 frame: 8 frame: 9 frame: 10

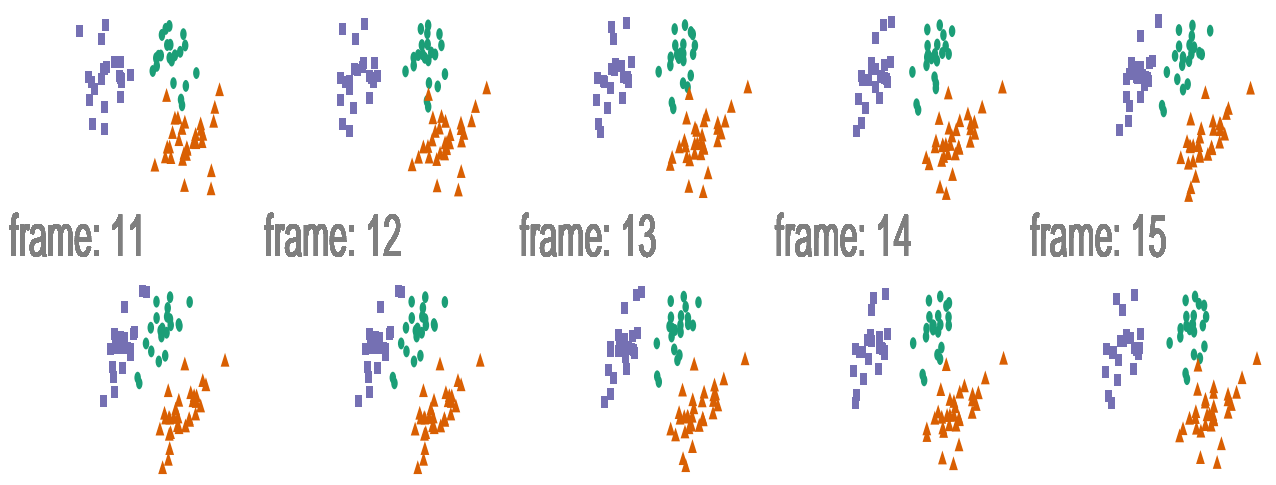

Figure 4: Radial manual tour manipulating aede2 of standardized flea data. The axis for aede2 increases in contribution to the projection, from its initial value to 1 , decreasing to 0 and then returning to the initial value. This effects the separation between the purple and green clusters. This shows that aede2 is important for distinguishing the purple species, because the separation disappears when aede 2 is not contributing to the projection. 


\section{Usage}

Using the flea data from the tourr package, we will illustrate generating a manual tour to explore the sensitivity of the cluster structure is to the variable aede2.

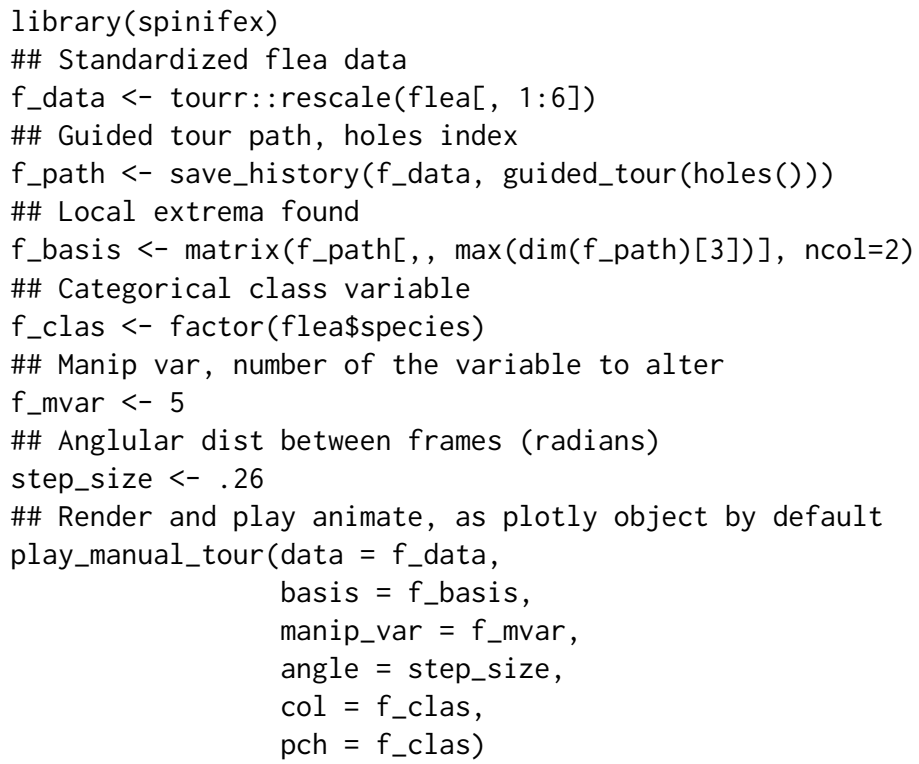

The play_manual_tour () function is a composite function handling interaction between manual_tour(), array $2 \mathrm{df}()$, and render_plotly(). This will generate an $\mathrm{html}$ animation using plotly. Switching the renderer to render_gganimate() alternatively creates an animated gif. Each of these formats allows for the animation to be made available on a web site, or directly visible in an html formatted document.

\section{Making illustrations}

The function oblique_frame can be used to show a projection of the basis, or with the data overlaid. For example, the plots in Figures 1 and 3 were made with code similar to this:

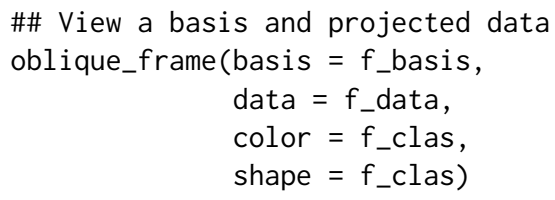

An illustration of the manip space (as shown in Figure 2) is made with the view_manip_space function, as follows:

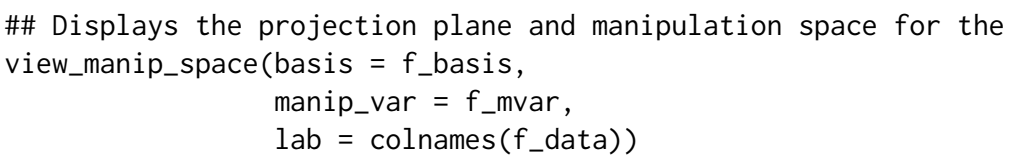

\section{Application}

Wang et al. (2018) introduces a new tool, PDFSense, to visualize the sensitivity of hadronic experiments to nucleon structure. The parameter-space of these experiments lies in 56 dimensions, $\delta \in \mathbb{R}^{56}$, and are visualized as 3D subspaces of the 10 first principal components in linear (PCA) and non-linear (t-SNE) embeddings.

Cook et al. (2018) illustrates how to learn more about the structures using a grand tour. Tours can better resolve the shape of clusters, intra-cluster detail, and better outlier detection than PDFSense \& TFEP (TensorFlow embedded projections) or traditional static embeddings. This example builds from here, illustrating how the manual tour can be used to examine the sensitivity of structure in a projection to different parameters. The specific $2 \mathrm{D}$ projections passed to the manual tour were provided in their work. 
The data has a hierarchical structure with top-level clusters; DIS, VBP, and jet. Each cluster is a particular class of experiments, each with many experimental datasets which, each have many observations of their own. In consideration of data density, we conduct manual tours on subsets of the DIS and jet clusters. This explores the sensitivity of the structure to each of the variables in turn and we present the subjectively best and worst variable to manipulate for identifying dimensionality of the clusters and describing the span of the clusters.

\section{Jet cluster}

The jet cluster resides in a smaller dimensionality than the full set of experiments with four principal components explaining 95\% of the variation in the cluster (Cook et al., 2018). The data within this 4D embedding is further subsetted, to ATLAS7old and ATLAS7new, to focus on two groups that occupy different parts of the subspace. Radial manual tours controlling contributions from PC4 and PC 3 are shown in Figures 5 and 6, respectively. The difference in shape can be interpreted as the experiments probing different phase-spaces. Back-transforming the principal components to the original variables can be done for a more detailed interpretation.

When PC4 is removed from the projection (Figure 5) the difference between the two groups is removed, indicating that it is important for distinguishing experiments. However, removing PC3 from the projection (Figure 6) does not affect the structure, indicating it is not important for distinguishing experiments. Animations for the remaining PCs can be viewed at the following links: PC1, PC2, PC3, and PC4. It can be seen that only PC4 is important for viewing the difference in these two experiments.

\section{DIS cluster}

Following Cook et al. (2018), to explore the DIS cluster, PCA is recomputed and the first six principal components, explaining $48 \%$ of the full sample variation, are used. The contributions of PC6 and PC2 are explored in Figures 7 and 8, respectively. Three experiments are examined: DIS HERA1+2 (green), dimuon SIDIS (purple), and charm SIDIS (orange).

Both PC2 and PC6 contribute to the projection similarly. When PC6 is rotated into the projection, variation in the DIS HERA1+2 is greatly reduced. When PC 2 is removed from the projection, dimuon SIDIS becomes more clearly distinct. Even though both variables contribute similarly to the original projection, their contributions have quite different effects on the structure of each cluster, and the distinction between clusters. Animations of all of the principal components can be viewed from the links: PC1, PC2, PC3, PC4, PC5, and PC6.

\section{Discussion}

Dynamic linear projections of numeric multivariate data, tours, play an important role in data visualization; they extend the dimensionality of visuals to peek into high-dimensional data and parameter spaces. This research has taken the manual tour algorithm, specifically the radial rotation, used in GGobi (Swayne et al., 2003-08-28) to interactively rotate a variable into or out of a 2D projection, and modified it to create an animation that performs the same task. It is most useful for examining the importance of variables, and how the structure in the projection is sensitive or not to specific variables. This functionality available in package spinifex. The work complements the methods available in the tourr package.

This work was motivated by problems in physics, and thus the usage was illustrated on data comparing experiments of hadronic collisions, to explore the sensitivity of cluster structure to different principal components. These tools can be applied quite broadly to many multivariate data analysis problems.

The manual tour is constrained in the sense that the effect of one variable is dependent on the contributions of other variables in the manip space. However, this can be useful to simplify a projection by removing variables without affecting the visible structure. Defining a manual rotation in high dimensions is possible using Givens rotations and Householder reflections as outlined in Buja et al. (2005). This would provide more flexible manual rotation, but more difficult for a user because they have the choice (too much choice) of which directions to move.

Another future research topic could be to extend the algorithm for use on 3D projections. With the current popularity and availability of $3 \mathrm{D}$ virtual displays, this may benefit the detection and understanding of the higher dimensional structure, or enable the examination of functions.

Having a graphical user interface would be useful for making it easier and more accessible to a general audience. This is possible to implement using shiny (Chang et al., 2020). The primary 


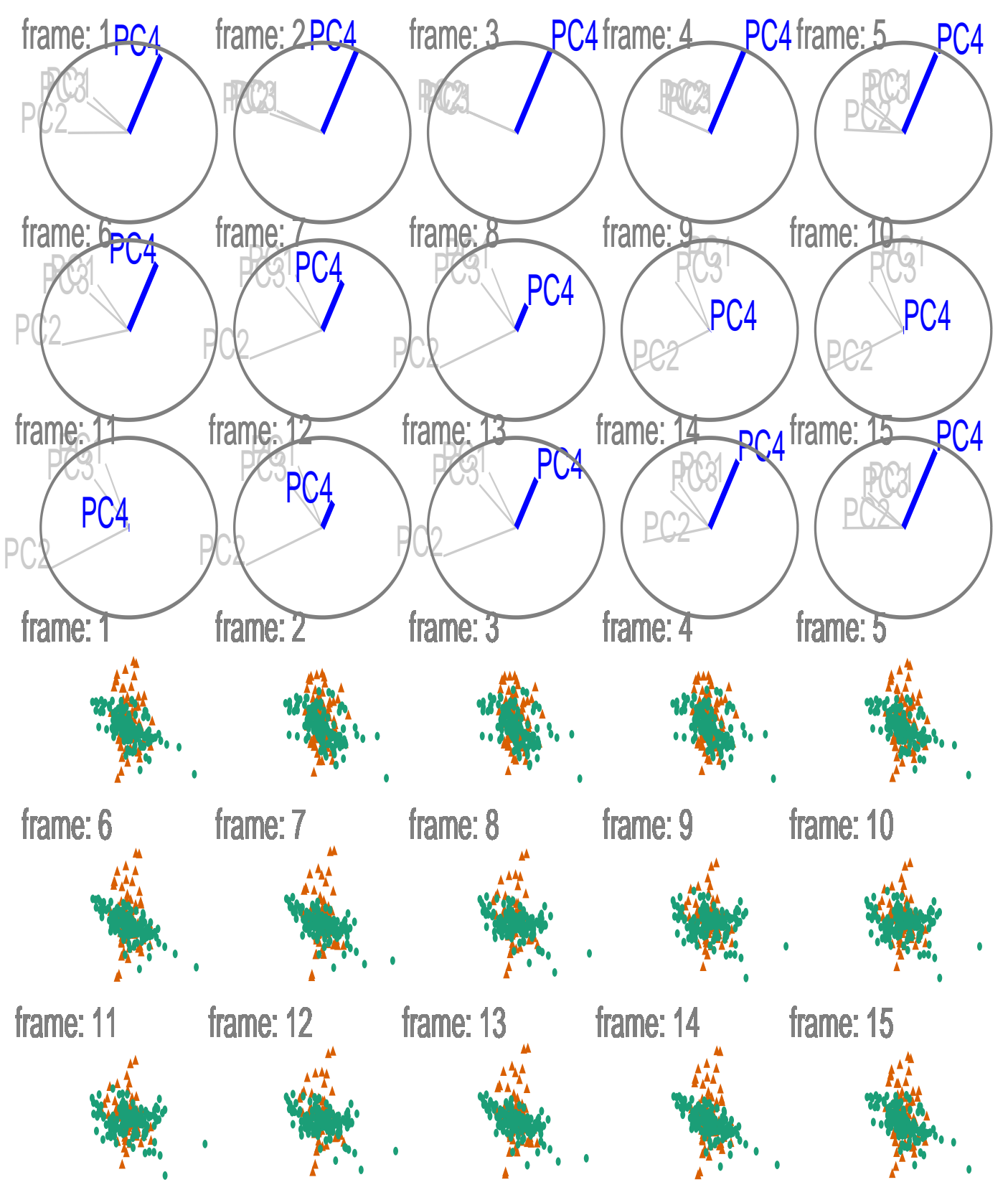

Figure 5: Snapshots of a radial manual tour of PC4 within the jet cluster, with color indicating experiment type: ATLAS7new (green) and ATLAS7old (orange). When PC4 is removed from the projection (frame 10) there is little difference between the groups, suggesting that PC4 is important for distinguishing the experiments. 


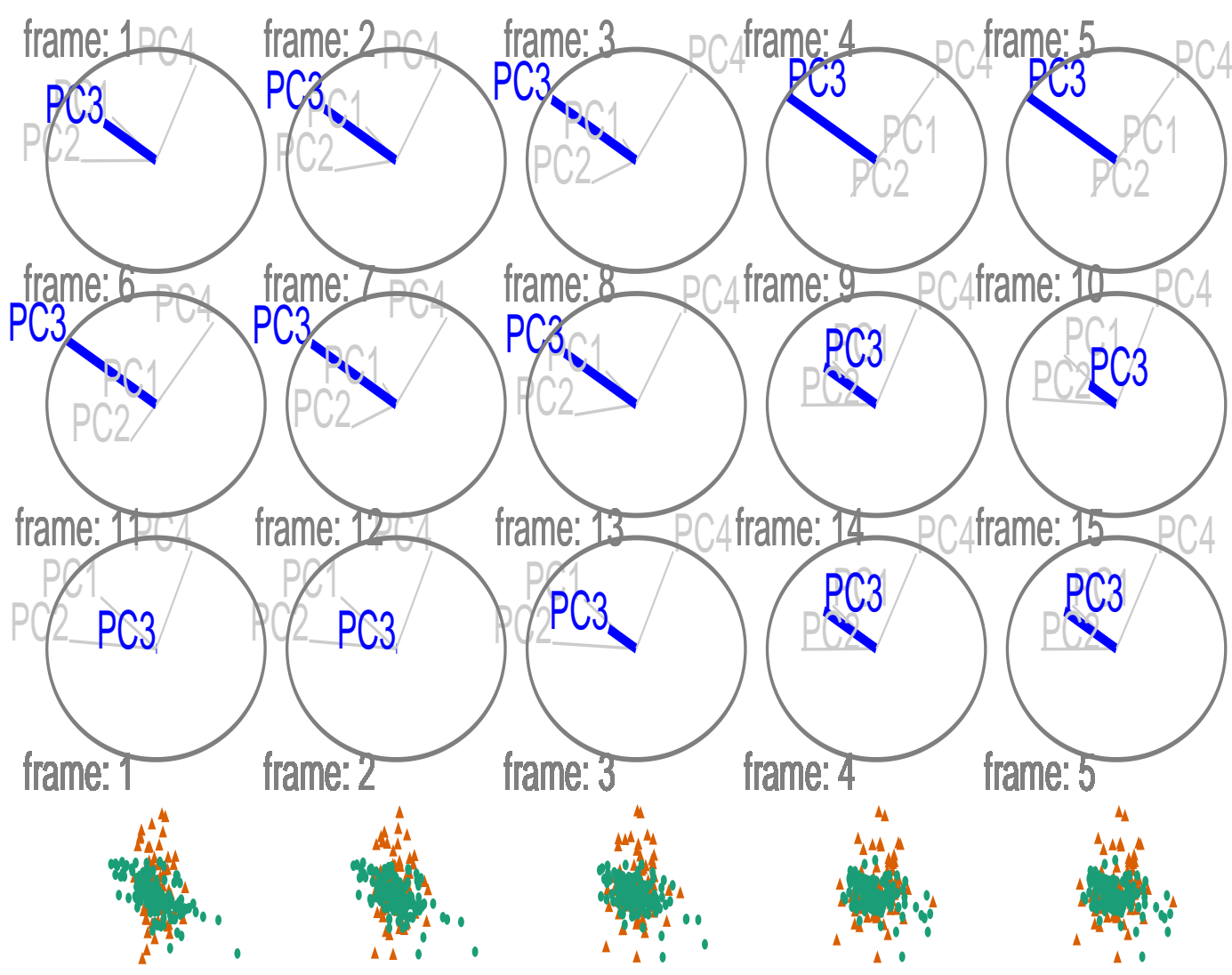

frame: 6

frame: 7

frame: 8

frame: 9

frame: 10
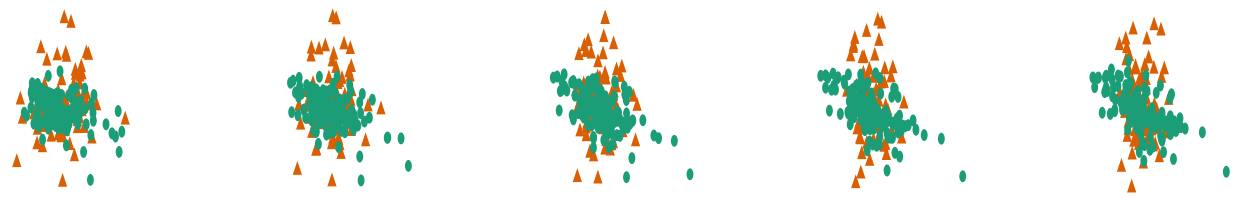

frame: 11

frame: 12

frame: 13

frame: 14

frame: 15
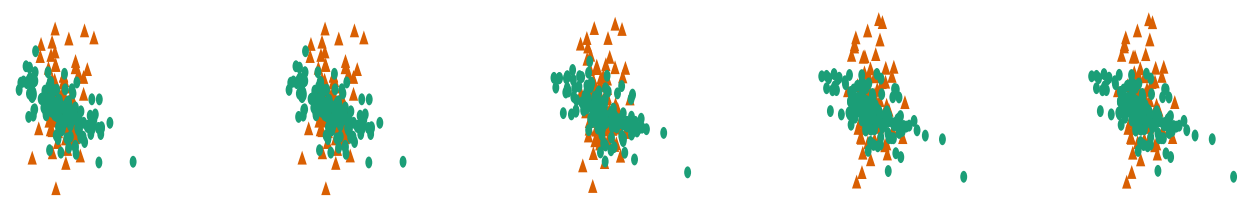

Figure 6: Snapshots of a radial manual tour of PC3 within the jet cluster, with color indicating experiment type: ATLAS7new (green) and ATLAS7old (orange). When the contribution from PC3 is changed there is little change to the structure of the two groups, suggesting that PC3 is not important for distinguishing the experiments. 


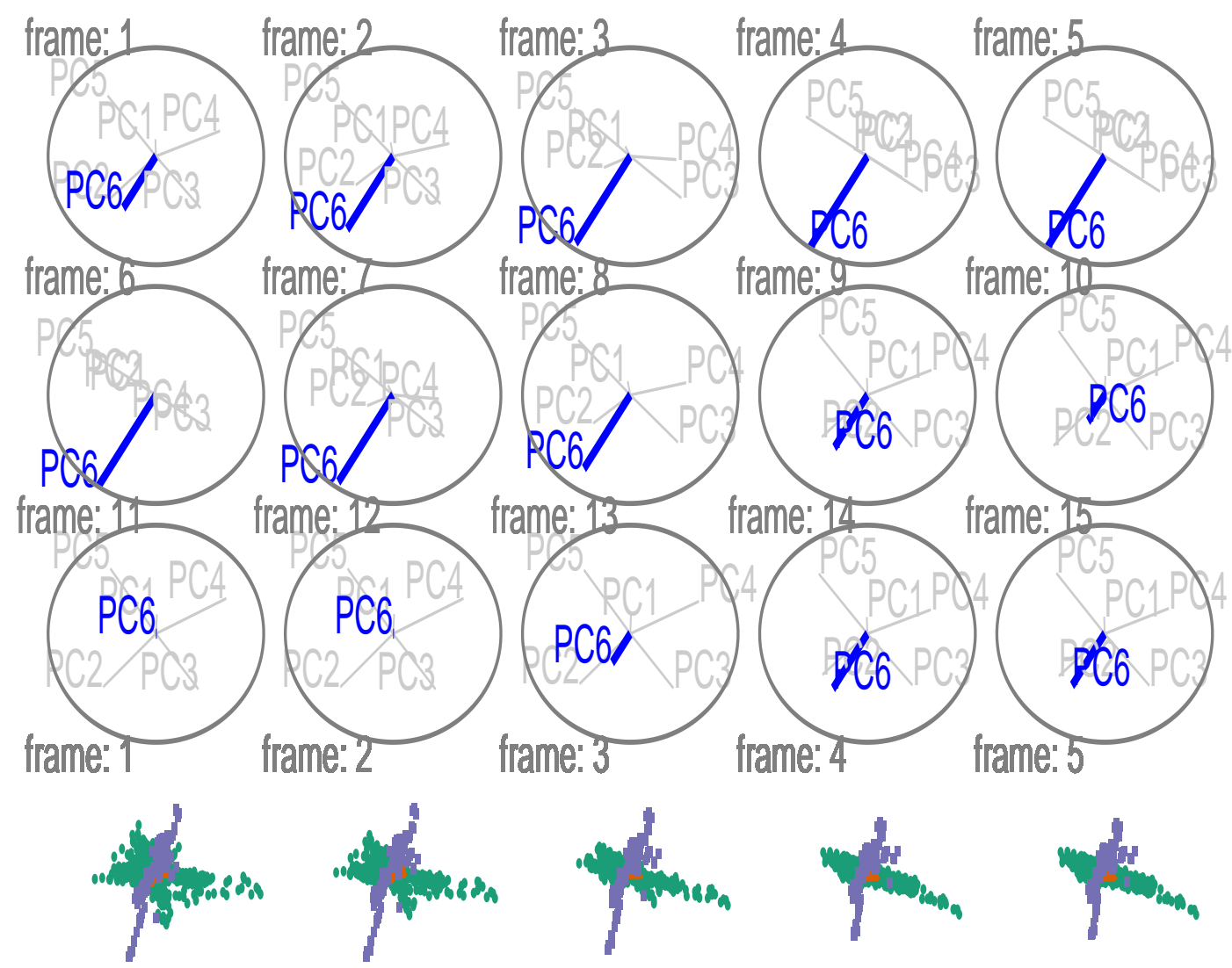

frame: 6

frame: 7

frame: 8

frame: 9 frame: 10
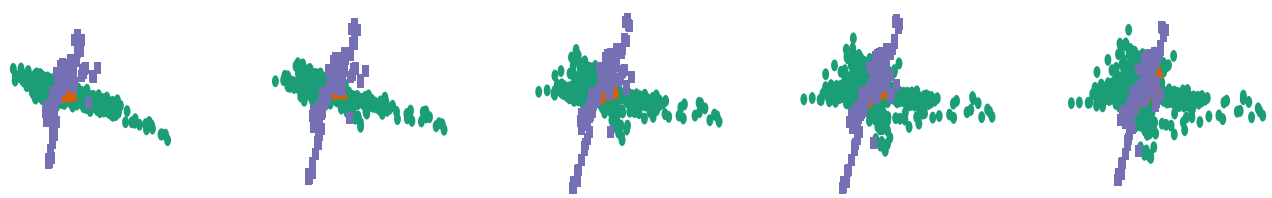

frame: 11

frame: 12

frame: 13

frame: 14

frame: 15

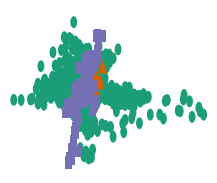

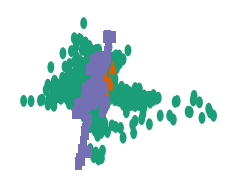

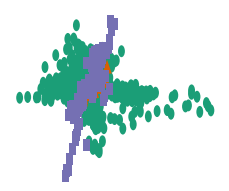

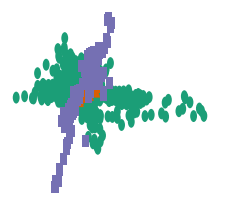

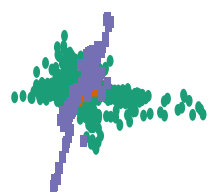

Figure 7: Snapshots of a radial manual tour exploring the sensitivity PC6 has on the structure of the DIS cluster, with color indicating experiment type: DIS HERA1+2 (green), dimuon SIDIS (purple), and charm SIDIS (orange). DIS HERA1+2 is distributed in a cross-shaped plane, charm SIDIS occupies the center of this cross, and dimuon SIDIS is a linear cluster crossing DIS HERA1+2. As the contribution of PC6 is increased, DIS HERA1+2 becomes almost singular in one direction (frame 5), indicating that this experiment has very little variability in the direction of PC6. 

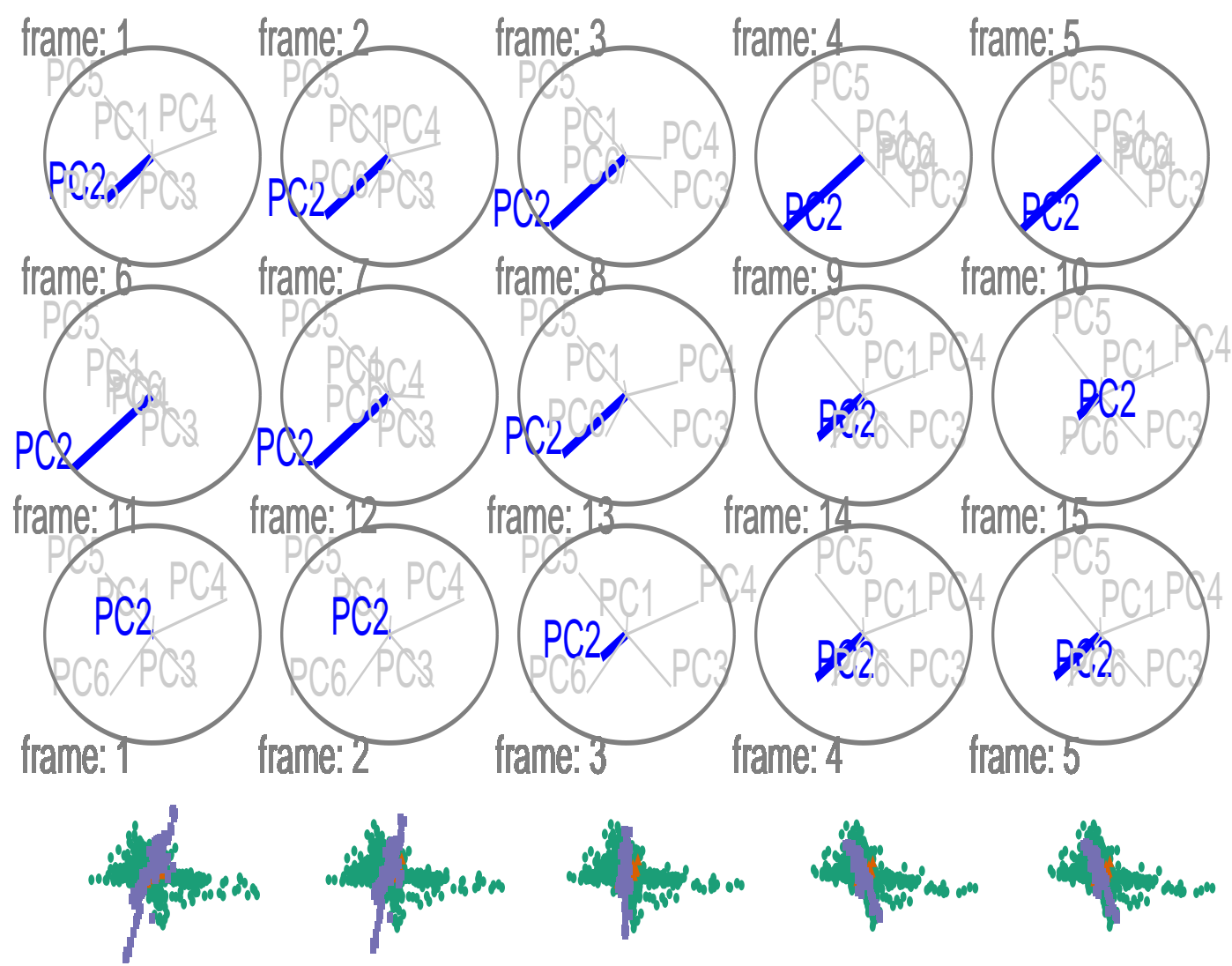

frame: 6

frame: 7

frame: 8

frame: 9

frame: 10
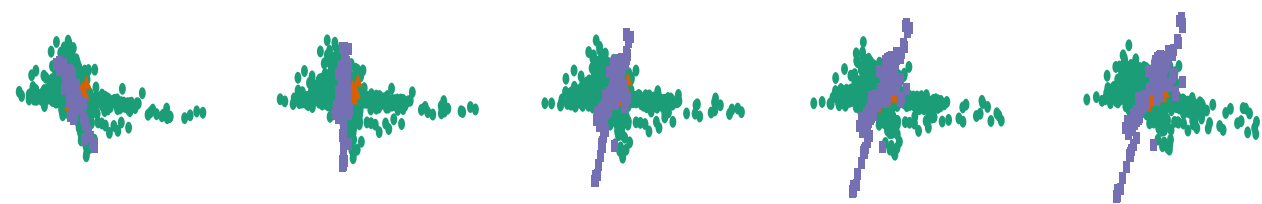

frame: 11

frame:. 12

frame.' 13

frame: 14

frame: 15

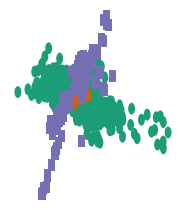

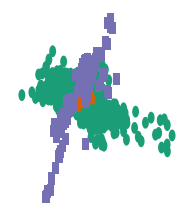

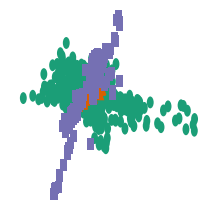

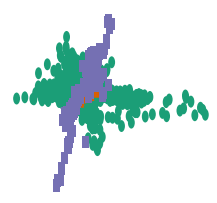

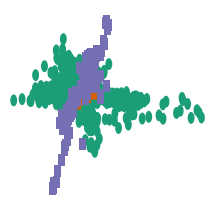

Figure 8: Snapshots of a radial manual tour exploring the sensitivity PC2 to the structure of the DIS cluster, with color indicating experiment type: DIS HERA1+2 (green), dimuon SIDIS (purple), and charm SIDIS (orange). As contribution from PC2 is decreased, dimuon SIDIS becomes more distinguishable from the other two clusters (frames 10-14), indicating that in its absence PC2 is important. 
purposes of the interface would be to allow the user to interactively change the manip variable easily, and the interpolation step for more or less detailed views.

\section{Acknowledgments}

This article was created in R, using knitr (Xie, 2020) and rmarkdown (Allaire et al., 2020), with code generating the examples inline. The source files for this article be found at github.com/nspyrison/spinifex_paper/. The animated gifs can also be viewed at this site, and also in the supplementary material for this paper. The source code for the spinifex package can be found at github.com/nspyrison/spinifex/.

\section{Bibliography}

J. J. Allaire, Y. Xie, J. McPherson, J. Luraschi, K. Ushey, A. Atkins, H. Wickham, J. Cheng, W. Chang, and R. Iannone. rmarkdown: Dynamic documents for r, 2020. URL https://github. com/rstudio/ rmarkdown. [p256]

D. Asimov. The grand tour: a tool for viewing multidimensional data. SIAM journal on scientific and statistical computing, 6(1):128-143, 1985. doi: https://doi.org/10.1137/0906011. [p243]

A. Buja, D. Cook, D. Asimov, and C. Hurley. Computational methods for high-dimensional rotations in data visualization. In Handbook of Statistics, volume 24, pages 391-413. Elsevier, 2005. ISBN 978-0-444-51141-6. doi: 10.1016/S0169-7161(04)24014-7. URL http://linkinghub elsevier. com/ retrieve/pii/S0169716104240147. [p251]

W. Chang, J. Cheng, J. J. Allaire, Y. Xie, and J. McPherson. shiny: Web application framework for r, 2020. URL https://CRAN. R-project.org/package=shiny. [p251]

D. Cook and A. Buja. Manual controls for high-dimensional data projections. Journal of Computational and Graphical Statistics, 6(4):464-480, 1997. ISSN 1061-8600. doi: 10.2307/1390747. URL http: //www. jstor. org/stable/1390747. [p243]

D. Cook, A. Buja, J. Cabrera, and C. Hurley. Grand tour and projection pursuit. Journal of Computational and Graphical Statistics, 4(3):155, 1995-09. ISSN 10618600. doi: 10.2307/1390844. URL https: //www. jstor. org/stable/1390844?origin=crossref. [p243]

D. Cook, U. Laa, and G. Valencia. Dynamical projections for the visualization of PDFSense data. Eur. Phys. J. C, 78(9):742, 2018. doi: 10.1140/epjc/s10052-018-6205-2. [p250, 251]

S. Kirkpatrick, C. D. Gelatt, and M. P. Vecchi. Optimization by simulated annealing. science, 220(4598): 671-680, 1983. doi: 10.1126/science.220.4598.671. [p243]

A. A. Lubischew. On the use of discriminant functions in taxonomy. Biometrics, pages 455-477, 1962. doi: 10.2307/2527894. [p244]

T. L. Pedersen and D. Robinson. gganimate: A grammar of animated graphics, 2020. URL https: //CRAN.R-project.org/package=gganimate. [p243]

O. Rodrigues. Des lois géométriques qui régissent les déplacements d'un système solide dans l'espace: et de la variation des cordonnées provenant de ces déplacements considérés indépendamment des causes qui peuvent les produire. Journal de Mathématiques Pures et Appliquées, 5:380-440, 1840. [p245]

C. Sievert. Interactive Web-Based Data Visualization with $R$, plotly, and shiny. Chapman and Hall/CRC, 2020. ISBN 978-1-138-33145-7. URL https://plotly-r.com. [p243]

D. F. Swayne, D. T. Lang, A. Buja, and D. Cook. GGobi: evolving from XGobi into an extensible framework for interactive data visualization. Computational Statistics E Data Analysis, 43(4):423-444, 2003-08-28. ISSN 0167-9473. doi: 10.1016/S0167-9473(02)00286-4. URL http: //www. sciencedirect. com/science/article/pii/s0167947302002864. [p251]

B.-T. Wang, T. J. Hobbs, S. Doyle, J. Gao, T.-J. Hou, P. M. Nadolsky, and F. I. Olness. Mapping the sensitivity of hadronic experiments to nucleon structure. Physical Review D, 98(9):094030, 2018. doi: 10.1103/PhysRevD.98.094030. [p243, 250]

H. Wickham. ggplot2: Elegant Graphics for Data Analysis. Springer-Verlag New York, 2016. ISBN 978-3-319-24277-4. URL https: //ggplot2. tidyverse.org. [p244] 
H. Wickham, D. Cook, H. Hofmann, and A. Buja. tourr: An r package for exploring multivariate data with projections. Journal of Statistical Software, 40(2), 2011. ISSN 1548-7660. doi: 10.18637/jss.v040.i02. URL http: //www. jstatsoft.org/v40/i02/. [p243]

Y. Xie. knitr: A general-purpose package for dynamic report generation in $r$, 2020. URL https: //yihui.org/knitr/. [p256]

Nicholas Spyrison

Monash University

Faculty of Information Technology

nicholas.spyrison@monash.edu

Dianne Cook

Monash University

Department of Econometrics and Business Statistics

dicook@monash.edu 